\title{
Nonsurgical Management of Periapical Lesion in Mandibular Incisors with 2 Canals - A Case Report
}

\author{
Shelly Sharma ${ }^{1}$, Anshul Arora² ${ }^{2}$ Mandeep S. Grewal ${ }^{3}$, Mamta Singla ${ }^{4}$, Lakshita Singh ${ }^{5}$ \\ 1, 2, 3, 4, 5 Department of Conservative Dentistry and Endodontics, \\ SGT University, Gurgaon, Haryana, India.
}

\section{INTRODUCTION}

In-depth knowledge of the root canal system is a major prerequisite for successful endodontic treatment. ${ }^{1}$ The major aim of endodontic treatment is the eradication of infection and prevention of reinfection in canal. However endodontic treatment may fail because of incomplete knowledge about the anatomical variation of root canals. Most of the times the canal remains untreated because of the inability of the dentist to recognize its presence. For good prognosis of the root canal treatment, proper exploration, complete debridement, biomechanical preparation, and filling of root canal system must be done. Therefore, a dentist must be familiar with all the various possible canal configurations. ${ }^{2}$ Many times, failure of endodontic treatment may occur because the morphological variation of the tooth unfavourably affects the treatment.

Pulpal inflammation can occur as a result of many factors like dental caries or trauma which causes tissue necrosis. Periapical tissue eradication develops in response to microbial accumulation and infiltration of their by-products in the periradicular tissues and activates host's immune reaction. ${ }^{3}$ The following case report presents the non-surgical management of mandibular central and lateral incisors, with each having two separate canals which join together to form a single canal just before exiting the apical foramen. Before starting root canal treatment, a careful radiographic examination should be done to detect the morphological variations in root canal anatomy. This paper describes two clinical cases of mandibular incisors with or without periapical lesion having two canals.

\section{PRESENTATION OF CASE}

A 40-year-old female patient reported with chief complaint of pain in mandibular anterior teeth region. Clinical examination revealed dental caries with respect to 32 and 41 and 32 were tender on vertical percussion. Radiographic examination showed periapical radiolucency with respect to 41 suggestive of chronic periapical abscess. [Figure 2(a)]

\section{DISCUSSION OF MANAGEMENT}

Non-surgical root canal treatment was planned with exploration, biomechanical preparation and obturation of these teeth. Isolation was done under rubber dam. Access cavity preparation was done through incisal edges initially with round bur and then with round end tapered bur with large labiolingual extension. [Figure 1(a), 2(a)] After endodontic exploration these teeth were found to have two canal orifices. Then after placing the file in both the canals a working length radiograph was taken which shows 2 canal orifices extending into two canals and joining short of the apex to continue as a single canal with single apical opening (Vertucci's Type II canal morphology.[Figure 1 (b), 2(b)]
Corresponding Author:

Dr. Anshul Arora,

Department of Conservative Dentistry and Endodontics,

Faculty of Dental Sciences, SGT University, Gurgaon, Haryana, India.

E-mail: anshularora2586@gmail.com

DOI: $10.14260 / j e m d s / 2021 / 476$

How to Cite This Article:

Sharma S, Arora A, Grewal MS, et al. Nonsurgical management of periapical lesion in mandibular incisors with 2 canals - a case report. J Evolution Med Dent Sci 2021;10(30):2331-2333, DOI: $10.14260 / j e m d s / 2021 / 476$

Submission 01-03-2021,

Peer Review 05-07-2021,

Acceptance 12-07-2021,

Published 26-07-2021.

Copyright @ 2021 Shelly Sharma et al. This is an open access article distributed under Creative Commons Attribution License [Attribution 4.0 International (CC BY 4.0)] 


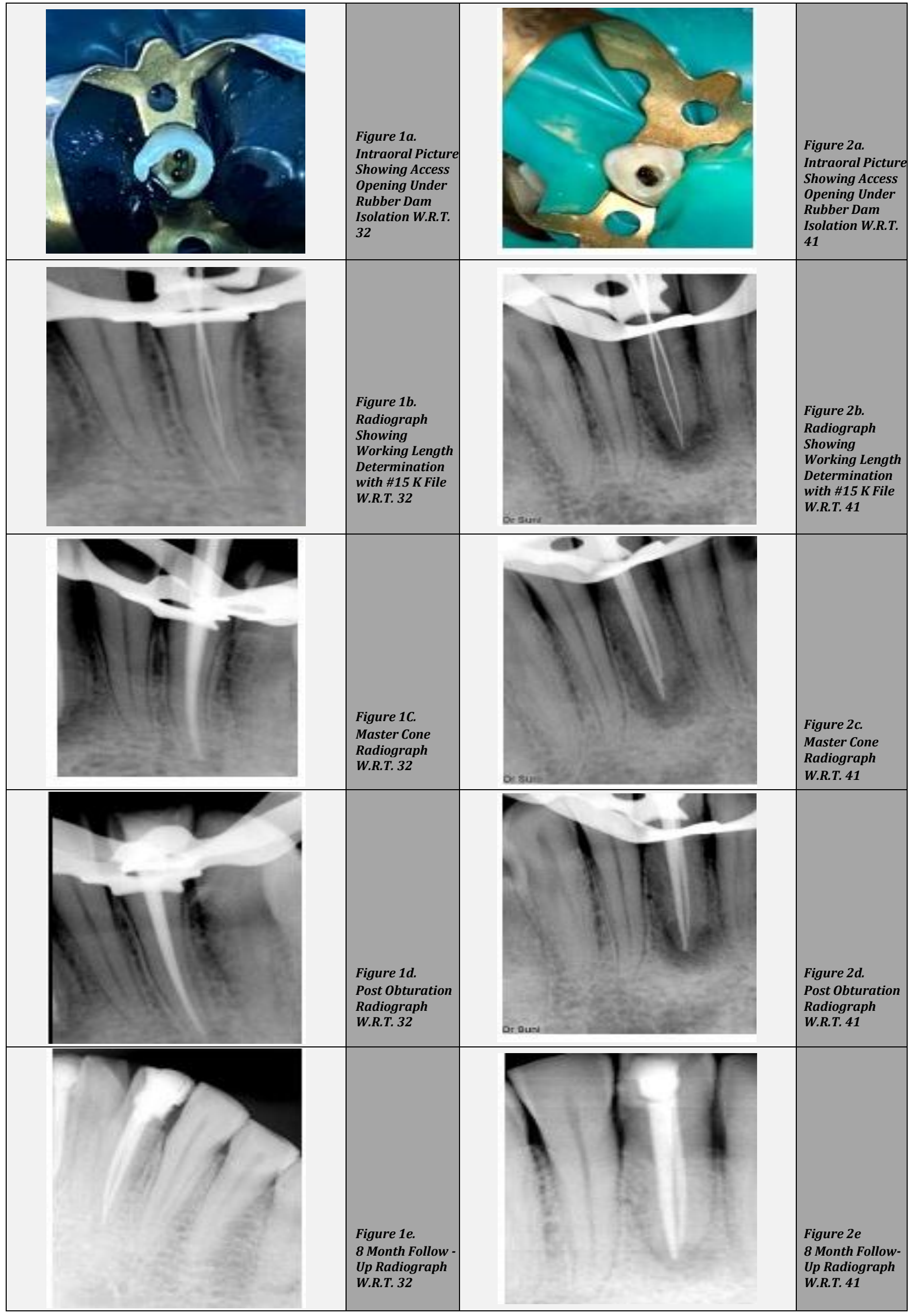


Biomechanical preparation was done by using M PRO Rotary file system and use of $5.25 \%$ sodium hypochlorite and $17 \%$ ethylenediamine tetra-acetic acid (EDTA) irrigation. Isolation was done, and the canals dried and then calcium hydroxide was placed as intracanal medicament for 1 week.

On the next appointment, all teeth were asymptomatic and obturation was done in both the cases with gutta percha points by using cold lateral condensation technique. [Figure 1(d), 2(d)]. Post-treatment intraoral periapical radiographs were taken with multiple angulations for better identification of 2 canals. After follow-up period of 8 months, IOPA showed complete periapical healing w. r. t 41. [figure2 (e)]

\section{DISCUSSION}

For successful endodontic treatment, a properly designed access cavity is of utmost importance. If access preparation is inadequate, instrumentation during biomechanical preparation becomes difficult in the canals with morphological variations. An adequate access cavity is necessary to establish straight line access to the apical foramen. ${ }^{4}$ The access cavity preparation of mandibular incisors is most crucial because of their small size. In most of the cases, mandibular anteriors have 2 canals, present in labiolingual direction. Out of these 2 canals, buccal canal is straight and easy to locate and lingual canal is most often missed because of lingual shelf. To avoid this, lingual shoulder should be removed completely by extending the access preparation well into cingulum gingival area, because if the second canal exists, it lies directly beneath it. Favourable outcome of endodontic treatment depends on the good preoperative radiograph, adequate debridement of the canals and proper sealing of root canal filling materials. ${ }^{5}$ It is recommended in literature that radiographic examination should be done from several different angles before access cavity preparation and clinician must always be on the watch for existence of second canal. In a study Klein et al. found that 20 degrees right and 30 degrees left horizontal beam angulations were significantly better at identifying twin canals than the ortho radial view. ${ }^{6}$

Vertucci in 1974 conducted a study on root canal morphology of 100 mandibular anterior teeth and identified 8 types of pulp space configurations in incisors. He found that 23 $\%$ of mandibular incisors had type 2 configuration with two separated canals exiting the pulp chamber and joining together as a single canal just $1 \mathrm{~mm}$ short of radiographic apex. ${ }^{1}$ Sert et al. in 2004 examined 1400 extracted mandibular permanent teeth and found that mandibular central incisors had 2 canals in $68 \%$ of the cases and mandibular lateral incisors had 2 canals in $63 \%$ of the cases. ${ }^{1}$ Numerous types of antimicrobial agents have been recommended as intracanal medicaments. ${ }^{5}$ In this case, calcium hydroxide was used as the intracanal medicament. Post treatment clinical and radiographic evaluation shows favourable treatment outcome in these cases. In this case report mandibular left lateral incisor presented with consistent width of periodontal ligament space after endodontic treatment while periapical lesion associated with the right central incisor is disappeared completely throughout the follow-up period of 8 months [Figure 1(e), 2(e)]. Thus, these cases demonstrate that with sufficient knowledge of root canal system, non-surgical root canal treatment can be a preferable treatment approach for teeth with morphological variations.

\section{CONCLUSIONS}

Endodontic treatment of mandibular incisors may fail because of inability to negotiate and manage the second canal. To avoid this, radiographic interpretation from multiple angulations is necessary. Mandibular incisors with 2 canals is a common entity as suggested by researchers. Thorough knowledge and labiolingual extension of access opening is needed to negotiate the multiple canals. The dentist should have a clear picture in mind about the number of canals to be expected for a particular tooth and their location.

Financial or other competing interests: None.

Disclosure forms provided by the authors are available with the full text of this article at jemds.com.

\section{REFERENCES}

[1] Shemesh A, Kavalerchik E, Levin A, et al. Root canal morphology evaluation of central and lateral mandibular incisors using cone-beam computed tomography in an Israeli population. J Endod 2018;44(1):51-5.

[2] Al-Fouzan KS, AlManee A, Jan J, et al. Incidence of two canals in extracted mandibularincisors teeth of Saudi Arabian samples. Saudi Endod J 2012;2(2):65-9.

[3] Moshari A, Vatanpour M, Esnaashari E, et al. Nonsurgical management of an extensive endodontic periapical lesion: a case report. Iran Endod J 2017;12(1):116-9.

[4] Cohen S, Hargreaves K. Pathways of the Pulp. 9 $9^{\text {th }}$ edn. Elsevier Mosby 2006.

[5] Tiku AM, Kalaskar RR, Damle SG. An unusual presentation of all the mandibular anterior teeth with two root canals-a case report. J Indian Soc Pedod Prev Dent 2005;23(4):204-6.

[6] Klein RMF, Blake SA, Nattress BR, et al. Evaluation of Xray beam angulation for successful twin canal identification inmandibular incisors. Int Endod J 1997;30(1):58-63. 